\title{
Three de novo $D D X 3 X$ variants associated with distinctive brain developmental abnormalities and brain tumor in intellectually disabled females
}

\author{
Marcello Scala $\mathbb{D}^{1} \cdot$ Annalaura Torella ${ }^{2,3} \cdot$ Mariasavina Severino $^{4} \cdot$ Giovanni Morana $^{4} \cdot$ Raffaele Castello $^{2}$. \\ Andrea Accogli ${ }^{1}$ - Antonio Verrico ${ }^{5}$ Maria Stella Vari ${ }^{6}$. Gerarda Cappuccio ${ }^{7}$ - Michele Pinelli $\mathbb{1}^{7}$. \\ Giuseppina Vitiello ${ }^{7}$ Gaetano Terrone ${ }^{7}$ - Alessandra D'Amico ${ }^{8}$. TUDP consortium • \\ Vincenzo Nigro $^{2,3} \cdot$ Valeria Capra $^{1}$
}

Received: 18 October 2018 / Revised: 8 March 2019 / Accepted: 19 March 2019 / Published online: 1 April 2019

(c) European Society of Human Genetics 2019

\begin{abstract}
De novo $D D X 3 X$ variants account for $1-3 \%$ of syndromic intellectual disability (ID) in females and have been occasionally reported in males. Furthermore, somatic $D D X 3 X$ variants occur in several aggressive cancers, including medulloblastoma. We report three unrelated females with severe ID, dysmorphic features, and a common brain malformative pattern characterized by malformations of cortical development, callosal dysgenesis, basal ganglia anomalies, and midbrainhindbrain malformations. A pilocytic astrocytoma was incidentally diagnosed in Patient 1 and trigonocephaly was found in Patient 2. With the use of family based whole exome sequencing (WES), we identified three distinct de novo variants in $D D X 3 X$. These findings expand the phenotypic spectrum of $D D X 3 X$-related disorders, demonstrating unique neuroradiological features resembling those of the tubulinopathies, and support a role for $D D X 3 X$ in neuronal development. Our observations further suggest a possible link between germline $D D X 3 X$ variants and cancer development.
\end{abstract}

\section{Introduction}

De novo $D D X 3 X$ variants have been recently reported in $1-$ $3 \%$ of females with unexplained intellectual disability (ID)

These authors contributed equally: Marcello Scala, Annalaura Torella Telethon Undiagnosed Diseases Program (TUDP) consortium

Supplementary information The online version of this article (https:// doi.org/10.1038/s41431-019-0392-7) contains supplementary material, which is available to authorized users.

Marcello Scala

marcelloscala87@gmail.com

1 Department of Neurosurgery, IRCCS Istituto Giannina Gaslini, Via Gerolamo Gaslini, 5, 16147 Genoa, Italy

2 Telethon Institute of Genetics and Medicine, Pozzuoli, Naples, Italy

3 Department of Precision Medicine, University of Campania 'Luigi Vanvitelli', Naples, Italy

4 Neuroradiology Unit, IRCCS Istituto Giannina Gaslini, Via Gerolamo Gaslini, 5, 16147 Genoa, Italy
$[1,2] . D D X 3 X$ maps to $\mathrm{Xp} 11.3-11.23$ and is ubiquitously transcribed in human tissues, escaping X-inactivation $[1,3]$. It encodes an ATP-dependent 'DEAD-box' RNA helicase involved in RNA processing through secondary structure remodeling, with a pivotal role in the regulation of gene expression, cell cycle control, innate immunity, and viral replication [4-6]. We present three unrelated females with ID, dysmorphic features, and a peculiar brain malformative pattern, thus expanding the phenotypic spectrum of $D D X 3 X$-related disorders.

5 Neuro-oncology Unit, IRCCS Istituto Giannina Gaslini, Via Gerolamo Gaslini, 5, 16147 Genoa, Italy

6 Pediatric Neurology and Muscular Diseases Unit, Department of Neurosciences, Rehabilitation, Ophthalmology, Genetics, Maternal and Child Health, University of Genoa, IRCCS Istituto Giannina Gaslini, Genoa 16147, Italy

7 Department of Translational Medicine, Federico II University, Naples, Italy

8 Department of Molecular Medicine and Medical Biotechnology, Federico II University, Naples, Italy 


\section{Patients and methods}

The study was approved by the Institutional Ethics Committee and written informed consent was obtained according to Telethon Undiagnosed Program guidelines. Whole exome sequencing (WES) was performed by using the Agilent SureSelect QXT Clinical Research Exome (Agilent Technologies, Santa Clara, CA, USA). Sequencing data were processed using an in-house software for the execution of the GATK Best Practices pipeline for WES variant analysis (see Supplementary Material). After filtering for population genetics, family segregation, and predicted impact on protein sequence, the best candidates for all probands were de novo variants in $D D X 3 X$. The identified variants were validated by Sanger sequencing in all members of the trios and submitted into the gene variant database LOVD at https://www.LOVD.nl/DDX3X (Patient IDs 00219101, 00219102, 00219099).

\section{Results}

\section{Clinical and neuroimaging findings}

Patient 1 was the second-born to unrelated healthy parents with a healthy son. Antenatal history revealed intrauterine growth retardation (IUGR) due to placental insufficiency. She was delivered at $30^{+5}$ weeks' gestation. Her birth weight (BW) was $890 \mathrm{~g}(-1.79 \mathrm{SD})$, length $38 \mathrm{~cm}(-0.66 \mathrm{SD})$, and occipito-frontal circumference (OFC) $27 \mathrm{~cm}$ (-0.65 SD). Neonatal course was uneventful. During childhood, global DD was noticed and she developed spastic tetraparesis and severe scoliosis. At the age of 8 years, she was incidentally diagnosed with a pilocytic astrocytoma (PA) (WHO grade I). Physical examination at 11 years of age revealed occipital plagiocephalywith normal OFC $(51.5 \mathrm{~cm},-0.96 \mathrm{SD})$, several dysmorphisms (Fig. 1a, panel a), absent speech, spastic tetraparesis, strabismus, and horizontal nystagmus.

Patient 2 was the only child of unrelated healthy parents. Prenatal history revealed IUGR, increased nuchal translucency, oligohydramnios, and ventriculomegaly. She was born at $34^{+2}$ weeks' gestation. Her BW was $1,550 \mathrm{~g}(-1.46$ $\mathrm{SD})$, length $38 \mathrm{~cm}(-2.5 \mathrm{SD})$, and OFC $29 \mathrm{~cm}(-1.39 \mathrm{SD})$. Neonatal course was complicated by diffuse hypotonia and feeding difficulties. Brain ultrasonography confirmed isolated ventriculomegaly. Electroencephalogram (EEG) showed slow waves in the right cerebral hemisphere. Bilateral sensorineural hearing loss was found and fundoscopic examination showed optic subatrophy, but visual evoked potentials were normal. At 2 years of age, she had severe ID/DD, dysmorphic features, and microcephaly (OFC $43.2 \mathrm{~cm},-3.13 \mathrm{SD}$ ) with prominent metopic bridge (Fig. 1a, panel b). Physical examination showed dysphagia, alternating esotropia, axial hypotonia, and generalized dystonia.

Patient 3 was the only daughter of non-consanguineous healthy parents. Pregnancy was complicated by IUGR due to placental insufficiency. She was delivered at 38 weeks' gestation. Her BW was $2,000 \mathrm{~g}(-2.50 \mathrm{SD})$ length $45 \mathrm{~cm}$ $(-1.83 \mathrm{SD})$, and $\mathrm{OFC} 30.5 \mathrm{~cm} \quad(-2.39 \mathrm{SD})$. Neonatal course was uneventful. At 12 months of age, she experienced prolonged akinetic seizures with motor arrest and hypotonia, requiring phenobarbital therapy. At 10 years of age, she was nonverbal and microcephalic (OFC $46 \mathrm{~cm}$, -4.90 SD). Physical examination showed dysmorphisms (Fig. 1a, panel c), truncal hypotonia, hand stereotypies, and scoliosis. Sleep EEG revealed low-voltage fast activity over polymicrogyric cortex.

Brain MRI studies revealed a similar malformative pattern in all patients (Fig. 1b), characterized by bilateral frontal and perysilvian polymicrogyria (Patients 1 and 3) and fronto-insular dysgyria (Patient 2), variable degrees of callosal hypo-dysgenesis, dysmorphic basal ganglia with indistinct anterior limbs of internal capsules, small olfactory bulbs, pontine and inferior vermis hypoplasia. White matter was globally reduced, especially at the level of ventral cingulum, with enlargement of lateral ventricles and peculiar temporal horn dilatation. Additional findings included an incidental solid mass in left cerebellar hemisphere in Patient 1 and trigonocephaly in Patient 2. DTI studies showed marked hypoplasia of the corpus callosum with prevalent posterior involvement, and reduced volume of the anterior limbs of the internal capsule and ventral cingulum. No aberrant course of the cortico-spinal tracts or other major white matter bundles was noted (Fig. 2 and Fig. 3 Supplementary Material).

\section{Molecular findings}

WES identified three distinct de novo heterozygous variants in DDX3X: c.1511G >A, p.(Gly504Glu) (Patient 1); c.1436_1439delinsTCTC, p.(Asp479Arg480delinsValSer) (Patient 2); c.641_643delTCA, p.(Ile214del)(Patient 3). Genomic coordinates refer to genome assembly hg19 and $D D X 3 X$ nucleotide and amino acid variants to RefSeq entries NM_001356.3and NP_001347.3, respectively. The variants identified in patients 1 and 2 have not been previously reported in the literature or in any public database (including COSMIC, Decipher, Clinvar, and gnomAD). As to p. (Ile214del), the frameshift mutation affecting the same residue c.641_643delTCAinsCC, p.(Ile214Thrfs*7) has been reported as 'pathogenic' in ClinVar. All variants were interpreted according to the American College of Medical Genetics and Genomics (ACMG) guidelines and were classified as class 5 (Mutation Analysis Supplementary Material). 
$\mathbf{A}$

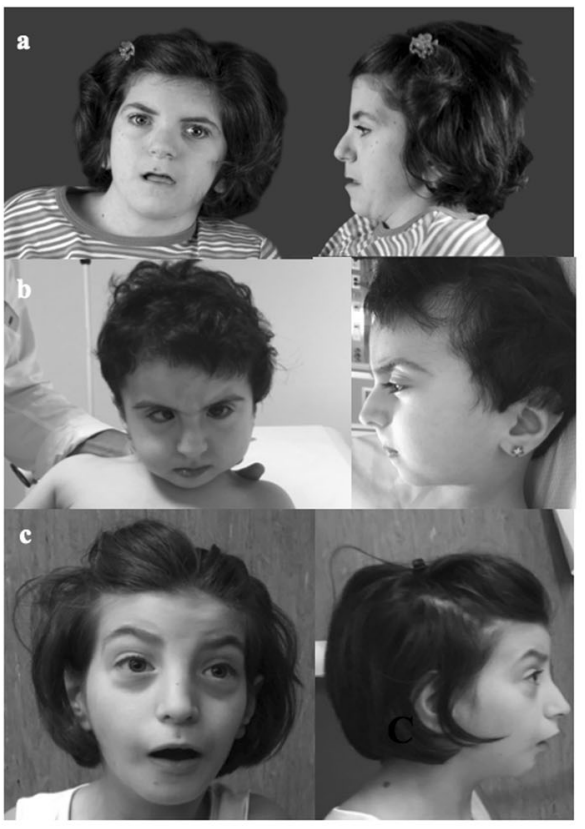

B

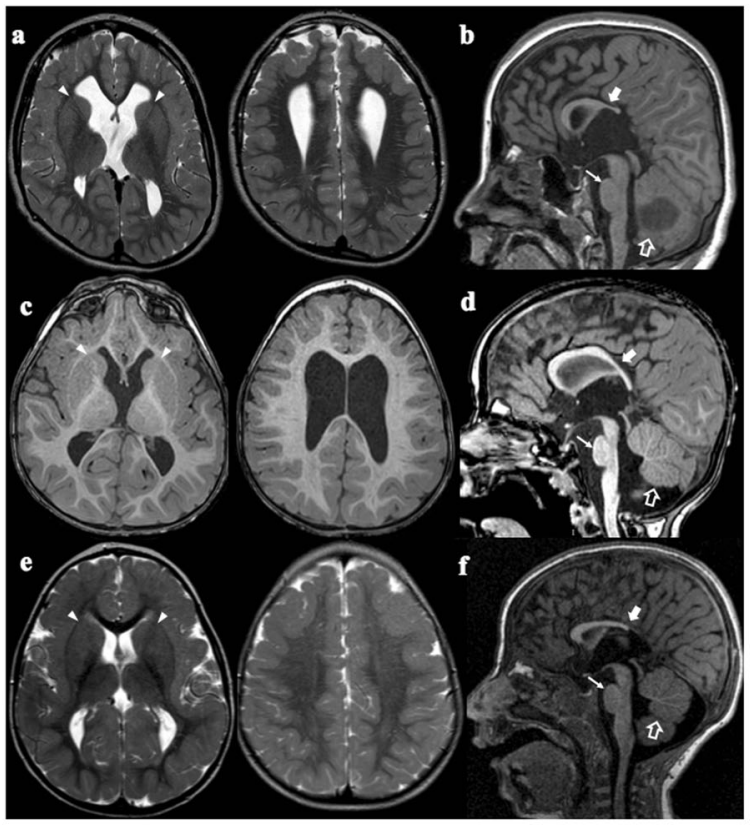

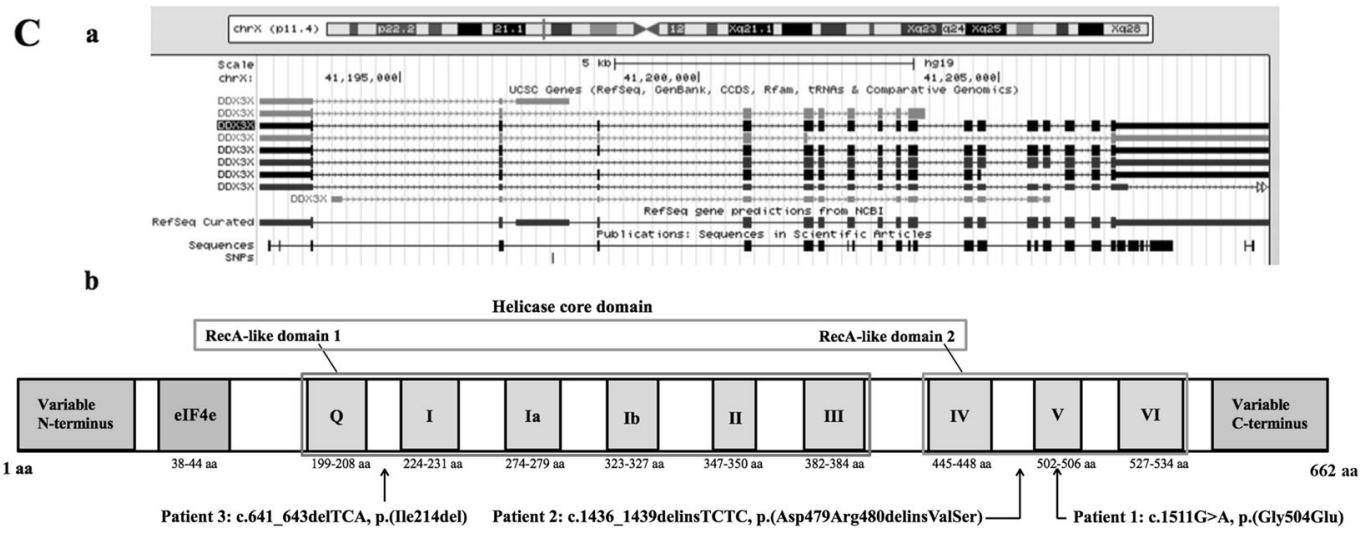

Fig. 1 a Dysmorphic facial features of the reported patients with de novo $D D X 3 X$ variants: a Patient 1 presents with occipital plagiocephaly, frizzy hair, flat face with midface hypoplasia, and short neck. Dysmorphic facial features include: arched eyebrows, mild esotropia of the left eye, low-set wide and simplified ears, long smooth philtrum, thin lips and upper vermillion border, absent cupid bow, and retrognathia; b Dysmorphic features of Patient 2 include prominent metopic ridge with glabellar nevus simplex, arched eyebrows with synophrys, hypotelorism with epicanthal folds, strabismus, low-set posteriorly rotated ears, malar hypoplasia, and microretrognathia; c Clinical photographs of Patient 3 showing arched eyebrows, midface hypoplasia, large ears, long philtrum, and retrognathia. b Brain MRI findings: a, b Patient 1 at the age of 8 years; c, d Patient 2 at the age of 2.5 years; e, f Patient 3 at the age of 12 years. In all patients, axial images reveal malformations of cortical development, characterized by bilateral frontal polymicrogyria in Patient 1 (a) and Patient 3 (b). In Patient 2, axial T1-weighted sequences show an abnormal fronto-insular gyral pattern (c). The anterior limb of the internal capsule is very small with dysmorphic appearance of the basal ganglia (a, c, d, arrowheads). The periventricular white matter is reduced, especially in Patients 1 and 2, with consequent enlargement of the lateral ventricles, especially in frontal and anterior temporal regions. Sagittal T1-weighted images demonstrate severe callosal hypodysgenesis with prevalent involvement of the isthmus and splenium in Patient 1 (b, thick arrow) and 3 (d, thick arrow), and milder callosal hypoplasia in Patient 2 (f, thick arrow). Note the marked hypoplasia of the anterior commissure in patient 1 and 2, and anterior commissure agenesis in Patient 3. There is pontine hypoplasia in all patients (arrows) associated with hypoplasia and mild rotation of the inferior portion of the vermis (open arrows, Patients 2 and 3). In patient 1 sagittal T1-weighted image demonstrates a hypointense cerebellar mass lesion (detailed depiction available in online Supplementary Fig. 1). c $D D X 3 X$ gene transcripts (top) and DDX3X protein (bottom): a UCSC genome browser map (GRCh37/hg19) shows multiple gene products of $D D X 3 X$, with alternative splicing resulting in multiple transcript variants. The gene has a $5^{\prime}-3^{\prime}$ orientation. The main transcript (black rectangle) of 16,874 bp is encoded by 17 exons; b Schematic, not to scale, representation of the domains of the full-length DDX3X protein and localization of the variants identified in the reported patients. N- and Cterminus are variable regions. The consensus eIF4E-binding sequence is important for the interaction with the eukaryotic initiation factor $4 \mathrm{E}$ (eIF4E), a translation initiation factor modulated by DDX3X. The RecA-like domains 1 and 2 are essential for the helicase activity. Each of these catalytic domains is composed of different conserved functional motifs, involved in ATP and RNA binding. a a amino acid 


\section{Discussion}

As of September 2018, 53 patients harboring $D D X 3 X$ variants have been collected in the Decipher database (Table 1). Most of the described variants are predicted to lead to loss of protein function (Table 2 Supplementary Material). Two large case series of 38 and 31 affected females harboring de novo or presumably de novo $D D X 3 X$ variants have been reported by Snijders Blok $L$ et al. and Wang et al., respectively [2, 7]. Conversely, only 10 male patients have been described so far, suggesting a lethal effect in most fetal cases [1, 7-9]. Six of them harbored maternally inherited missense variants, two carried de novo missense variants, and one carried a de novo splice site variant. The mode of inheritance in one patient harboring a missense variant was not confirmed [1, 7-9]. Regardless of gender, most $D D X 3 X$ patients carry missense variants affecting the ATP-binding or C-terminal helicase domains, similarly to those identified in our patients, without a clear genotype-phenotype correlation $[2,7,10]$.

$D D X 3 X$-related disorders are characterized by a relevant phenotypic variability (Table 1), especially with regard to the severity of neurodevelopmental involvement. While DD and/or ID are present in the vast majority of patients, a variable proportion of affected individuals show movement disorders, significant behavioral abnormalities, hypotonia, and seizures $[2,7]$. Facial dysmorphisms are common, including clinical features of Toriello-Carey syndrome [2, 7 , 8]. Interestingly, the existence of sex-specific phenotypes has been hypothesized [9]. Although all reported male patients have ID and global DD, and most of them suffer from movement disorders, they exhibit peculiar phenotypic features. In particular, male patients are rarely hypotonic as compared to females and often show craniofacial deformities (especially brachycephaly and plagiocephaly), ophthalmological abnormalities, and congenital heart disease [9].

In line with previous reports, our patients showed severe ID/DD, neurological abnormalities, microcephaly, and dysmorphic features (Table 3 Supplementary Material) [1]. Interestingly, occipital plagiocephaly was observed in Patient 1 and Patient 2 was diagnosed with sensorineural hearing loss and trigonocephaly. However, a true craniosynostosis was radiologically confirmed only in the latter (Fig. 3 Supplementary Material). While hearing impairment has been occasionally associated with $D D X 3 X$ spectrum, trigonocephaly has not been reported in patients with $D D X 3 X$-related ID so far $[2,8]$.

All patients displayed a common brain malformative pattern characterized by bilateral frontal and perysilvian polymicrogyria and/or dysgyria, callosal hypo-dysgenesis, dysmorphic basal ganglia with indistinct anterior limbs of internal capsules, hypoplasia of the ventral cingulum, incomplete hippocampal rotation, and pontine and inferior vermis hypoplasia. Although abnormal neuroradiological findings have been described in several females harboring $D D X 3 X$ variants, malformations of the cortical development have been only occasionally reported [1]. In particular, polymicrogyria was found in 4 out of 37 individuals of the largest case series published by Blok et al., and simplified gyral pattern was present in one patient reported by Dikow et al. [2, 8]. Remarkably, no mention of associated midbrain-hindbrain anomalies, basal ganglia dysmorphisms, and/or marked hypoplasia of ventral cingulum was made in these case series.

The neuroradiological features observed in our patients are similar, though less severe, to those reported in individuals with tubulinopathies or GRIN2B encephalopathy [11, 12]. These findings suggest that $D D X 3 X$ might play a pivotal role not only in neuronal proliferation and migration, but also in white matter development. Despite the presence of callosal abnormalities and pontine hypoplasia, we did not find aberrant white matter bundles on DTI tractography, thus ruling out major axonal pathfinding defects. DDX $3 X$ is required for neurite outgrowth and dendritic spine formation through the translational activation of mRNAs involved in Rac1 activation [13-15]. This would lead to speculate about a common underlying pathogenic mechanism for brain malformations in tubulinopathies and $D D X 3 X$-related disorders, possibly involving altered microtubule stability and defective migration of cortical GABAergic interneurons due to impaired Rac1 signaling [14]. However, the presence of similar brain malformations in a minority of patients with $D D X 3 X$ missense variants suggests a possible genotype-phenotype correlation requiring further validation in larger studies.

The incidental diagnosis of a cerebellar PA in Patient 1 represents another relevant aspect of this study. DDX3X plays a crucial role in cell cycle progression and is involved in $\mathrm{Wnt} / \beta$-catenin signaling pathway and cancer dissemination $[3,5,16]$. Somatic $D D X 3 X$ variants have been reported in several aggressive tumors, including Wnt-driven medulloblastoma $[3,5,17]$. Furthermore, a significant correlation between $D D X 3 X$ expression and Snail levels has been reported in glioblastoma multiforme [16]. The missense variant p.(Gly504Glu) identified in Patient 1 affects the central helicase core of DDX3X (Fig. 1c, panel b), similarly to the somatic variants occurring in medulloblastoma. These variants impair DDX3X-RNA binding resulting in altered protein function, which might play a relevant role in 'DDX3X-driven cancerogenesis' [3]. Accordingly, we speculate that the p.(Gly504Glu) variant might have contributed to PA development as part of a more complex pathogenic mechanism. However, obvious limitations are worth noting. First, functional studies on PA tissues were not performed. Furthermore, we did not seek a second hit in 
Table 1 Summary of the clinical and neuroradiological features of the Decipher patients with $D D X 3 X$ variants

\begin{tabular}{|c|c|c|}
\hline & Number & Total $(\%)$ \\
\hline \multicolumn{3}{|l|}{ Sex } \\
\hline Female & $50 / 53$ & 94.3 \\
\hline Male & $3 / 53$ & 5.7 \\
\hline Dysmorphic facial features present in $\geq 2$ PTS & $33 / 53 \mathrm{pts}$ & 62.3 \\
\hline Long/abnormal philtrum & 4 & \\
\hline Dental anomalies & 4 & \\
\hline Large ears/earlobes & 3 & \\
\hline Prominent nose & 3 & \\
\hline Narrow mouth & 3 & \\
\hline Thin vermillion border & 3 & \\
\hline High/narrow palate & 3 & \\
\hline Small face & 2 & \\
\hline Protruding ears & 2 & \\
\hline Wide/depressed nasal bridge & 2 & \\
\hline Thin upper lip vermillion & 2 & \\
\hline Protruding tongue & 2 & \\
\hline \multicolumn{3}{|l|}{ Additional clinical findings } \\
\hline Microcephaly & $18 / 53$ & 34 \\
\hline \multicolumn{3}{|l|}{ Skull abnormalities } \\
\hline Plagiocephaly & 2 & \\
\hline Brachycephaly & 1 & \\
\hline Skull asymmetry & 1 & \\
\hline Prominent metopic ridge & 1 & \\
\hline \multicolumn{3}{|l|}{ Skeletal/extremities } \\
\hline Hypermobility/laxity & 12 & \\
\hline Scoliosis & 4 & \\
\hline Short stature & 3 & \\
\hline Long fingers/toes & 3 & \\
\hline Limited elbow extension & 2 & \\
\hline Broad hallux/thumbs & 2 & \\
\hline Prominent fingerpads & 2 & \\
\hline \multicolumn{3}{|l|}{ Eyes } \\
\hline Strabismus; proptosis & $8 ; 1$ & \\
\hline Visual impairment & 3 & \\
\hline Unspecified eye anomalies & 2 & \\
\hline Optic nerve hypo/aplasia & 1 & \\
\hline Pigmentary retinopathy; retinal arteries tortuosity & $1 ; 1$ & \\
\hline \multicolumn{3}{|l|}{ Hearing impairment } \\
\hline Mixed & 2 & \\
\hline Conductive & 1 & \\
\hline Sensorineural & 1 & \\
\hline \multicolumn{3}{|l|}{ Cardiovascular and respiratory } \\
\hline Pulmonic stenosis & 3 & \\
\hline Tetralogy of Fallot & 1 & \\
\hline APW; EA; ASD & $1 ; 1 ; 1$ & \\
\hline Abnormal upper respiratory tract & 1 & \\
\hline \multicolumn{3}{|l|}{ Endocrine/GI tract: } \\
\hline Obesity & 8 (6 truncal) & \\
\hline Gastroesophageal reflux/esophagitis & 3 & \\
\hline Ectopic anus & 2 & \\
\hline Feeding difficulties; polyphagia & $1 ; 1$ & \\
\hline Umbilical hernia (umbilical; inguinal) & $1 ; 1$ & \\
\hline \multicolumn{3}{|l|}{ GU tract } \\
\hline Ovarian cysts & 3 & \\
\hline Vescicoureteral reflux & 1 & \\
\hline Multicystic kidney dysplasia & 1 & \\
\hline
\end{tabular}

Table 1 (continued)

\begin{tabular}{|c|c|c|}
\hline & Number & Total $(\%)$ \\
\hline \multicolumn{3}{|l|}{ Skin } \\
\hline $\begin{array}{l}\text { Skin nevi (linear sebaceous; congenital } \\
\text { melanocytic) }\end{array}$ & $4(2 ; 1)$ & \\
\hline Supernumerary nipples & 4 & \\
\hline Hypo/hyperpigmentation & 3 & \\
\hline Neurological abnormalities & $53 / 53$ & 100 \\
\hline ID & $12 / 53$ & 22.6 \\
\hline Severe & 9 & \\
\hline Moderate & 1 & \\
\hline Unspecified & 2 & \\
\hline Specific learning disabilities & $3 / 53$ & 5.6 \\
\hline $\mathrm{DD}$ & $33 / 53$ & 62.3 \\
\hline \multicolumn{3}{|l|}{ Severity: } \\
\hline Severe & 4 & \\
\hline Moderate & 10 & \\
\hline Mild & 2 & \\
\hline \multicolumn{3}{|l|}{ Type: } \\
\hline Global & 29 & \\
\hline Speech & 12 & \\
\hline Motor & 5 & \\
\hline Developmental regression & $1 / 53$ & 1.8 \\
\hline Behavioral abnormalities & $4 / 53$ & 7.5 \\
\hline Motor stereotypies & $\begin{array}{l}8 \text { ( } 7 \text { hand } \\
\text { flapping, } 1 \\
\text { unspecified) }\end{array}$ & \\
\hline Inappropriate laughter/ happy disposition & 3 & \\
\hline Hyperactivity/short attention span & 2 & \\
\hline Autistic spectrum & 2 & \\
\hline Sleep-wake disturbance & 2 & \\
\hline Self-injury & 1 & \\
\hline Unspecified & 1 & \\
\hline \multicolumn{3}{|l|}{ Common neurological features } \\
\hline Hypotonia; abnormal muscle tone & 7 (1 neonatal); 3 & 13.2 \\
\hline Ataxia & 7 & 13.2 \\
\hline Poor motor coordination; tip-toe gait & $2 ; 1$ & \\
\hline Drooling & 2 & \\
\hline Poor eye contact & 1 & 0 \\
\hline Brisk reflexes & 1 & 0 \\
\hline Dysarthria & 1 & 0 \\
\hline Seizures & $6 / 53$ & 11.3 \\
\hline Unspecified & 3 & 0 \\
\hline Focal & 2 & 0 \\
\hline Generalized & 1 & 0 \\
\hline Abnormal brain MRI findings & $16 / 53$ & 30.2 \\
\hline Abnormalities of CC & $7 / 53$ & 13.2 \\
\hline Hypoplasia & 3 & 0 \\
\hline Agenesis & 3 & \\
\hline Unspecified & 1 & \\
\hline Delayed CNS myelination & 3 & 5.6 \\
\hline Ventricular enlargement & 2 & \\
\hline Cerebellar anomalies & 2 & \\
\hline Hypoplasia & 1 & \\
\hline Abnormal white matter & 1 & \\
\hline Unspecified CNS abnormality & 2 & \\
\hline Cortical dysplasia & 1 & \\
\hline Stroke & 1 & \\
\hline
\end{tabular}

$A P W$ aortopulmonary window, $A S D$ atrial septal defect, $C C$ corpus callosum, $C N S$ central nervous system, $D D$ developmental delay, $E A$ Ebstein's anomaly, $G I$ gastrointestinal, $G U$ genitourinary, $I D$ intellectual disability, MRI magnetic resonance imaging, PTS patients. 
$D D X 3 X$ or further genes involved in tumorigenesis. Indeed, since $D D X 3 X$ exerts tumor suppressing functions, patients harboring $D D X 3 X$ germline variants might have a greater risk of developing somatic second hit mutations, leading to a possible increase in cancer risk [18].

In conclusion, this report expands the phenotypic spectrum of $D D X 3 X$-related disorders. Our findings suggest novel consequences of $D D X 3 X$ variants, resulting in altered neuronal migration and proliferation, abnormal white matter development, and possible promotion of tumorigenesis. Accordingly, $D D X 3 X$ should be considered in the differential diagnosis of tubulinopathies and included in the "cortical malformations" Next Generation Sequencing (NGS) panel. However, in the absence of pathognomonic clinical and radiological features due to the significant phenotypic variability, additional $D D X 3 X$ cases are expected to be detected by WES alone in the next future. Further studies will help clarify the actual clinical relevance and implications of these observations.

Acknowledgements We thank the patients and their families for the collaboration and the consent to the publication of this paper.

\section{Compliance with ethical standards}

Conflict of interest The authors declare that they have no conflict of interest.

Ethical approval This study was approved by the Ethical Committees of IRCCS Istituto Giannina Gaslini and Federico II University.

Informed consent Written informed consent was obtained from the parents of the subjects of the study.

Publisher's note: Springer Nature remains neutral with regard to jurisdictional claims in published maps and institutional affiliations.

\section{References}

1. Kellaris G, Khan K, Baig SM, Tsai IC, Zamora FM, Ruggieri P, et al. A hypomorphic inherited pathogenic variant in $D D X 3 X$ causes male intellectual disability with additional neurodevelopmental and neurodegenerative features. Hum Genomics. 2018;12:11.

2. Snijders Blok L, Madsen E, Juusola J, Gilissen C, Baralle D, Reijnders MR, et al. Mutations in $D D X 3 X$ are a common cause of unexplained intellectual disability with gender-specific effects on Wnt signaling. Am J Hum Genet. 2015;97:343-52.
3. Bol GM, Xie M, Raman V. DDX3, a potential target for cancer treatment. Mol Cancer. 2015;14:188.

4. Linder P, Lasko PF, Ashburner M, Leroy P, Nielsen PJ, Nishi K, et al. Birth of the D-E-A-D box. Nature. 1989;337:121-2.

5. Epling LB, Grace CR, Lowe BR, Partridge JF, Enemark EJ. Cancer-associated mutants of RNA helicase DDX3X are defective in RNA-stimulated ATP hydrolysis. J Mol Biol. 2015; 427:1779-96.

6. Schröder M. Human DEAD-box protein 3 has multiple functions in gene regulation and cell cycle control and is a prime target for viral manipulation. Biochem Pharmacol. 2010;79:297-306.

7. Wang X, Posey JE, Rosenfeld JA, Bacino CA, Scaglia F, Immken $\mathrm{L}$, et al. Phenotypic expansion in $D D X 3 X$ - a common cause of intellectual disability in females. Ann Clin Transl Neurol. 2018; 5:1277-85.

8. Dikow N, Granzow M, Graul-Neumann LM, Karch S, Hinderhofer $\mathrm{K}$, Paramasivam N, et al. $D D X 3 X$ mutations in two girls with a phenotype overlapping Toriello-Carey syndrome. Am J Med Genet A. 2017;173:1369-73.

9. Nicola P, Blackburn PR, Rasmussen KJ, Bertsch NL, Klee EW, Hasadsri L, et al. De novo DDX3X missense variants in males appear viable and contribute to syndromic intellectual disability. Am J Med Genet A. 2019 Feb 7. [Epub ahead of print]

10. Fieremans N, Van Esch H, Holvoet M, Van Goethem G, Devriendt $\mathrm{K}$, Rosello $\mathrm{M}$, et al. Identification of intellectual disability genes in female patients with a skewed X-inactivation pattern. Hum Mutat. 2016;37:804-11.

11. Mutch CA, Poduri A, Sahin M, Barry B, Walsh CA, Barkovich AJ. Disorders of microtubule function in neurons: imaging correlates. Am J Neuroradiol. 2016;37:528-35.

12. Platzer K, Yuan H, Schütz H, Winschel A, Chen W, Hu C, et al. GRIN2B encephalopathy: novel findings on phenotype, variant clustering, functional consequences and treatment aspects. J Med Genet. 2017;54:460-70.

13. Chen HH, Yu HI, Tarn WY. DDX3 modulates neurite development via translationally activating an RNA regulon involved in Rac1 activation. J Neurosci. 2016;36:9792-804.

14. Tivodar S, Kalemaki K, Kounoupa Z, Vidaki M, Theodorakis K, Denaxa M, et al. Rac-GTPases regulate microtubule stability and axon growth of cortical GABAergic interneurons. Cereb Cortex. 2015;25:2370-82.

15. Zamboni V, Armentano M, Berto G, Ciraolo E, Ghigo A, Garzotto D, et al. Hyperactivity of Rac1-GTPase pathway impairs neuritogenesis of cortical neurons by altering actin dynamics. Sci Rep. 2018;8:7254.

16. Sun M, Song L, Zhou T, Gillespie GY, Jope RS. The role of DDX3 in regulating Snail. Biochim Biophys Acta. 2011;1813:438-47.

17. Pugh TJ, Weeraratne SD, Archer TC, Pomeranz Krummel DA, Auclair D, Bochicchio J, et al. Medulloblastoma exome sequencing uncovers subtype-specific somatic mutations. Nature. 2012; 488:106-10.

18. Dunford A, Weinstock DM, Savova V, Schumacher SE, Cleary JP, Yoda A, et al. Tumor-suppressor genes that escape from Xinactivation contribute to cancer sex bias. Nat Genet. 2017;49:10-6. 